\title{
TERAPIAS NÃO FARMACOLÓGICAS UTILIZADAS PARA ALÍVIO DA DISMENORREIA PRIMÁRIA: REVISÃO INTEGRATIVA
}

\author{
NON-PHARMACOLOGICAL THERAPIES USED TO RELIEVE PRIMARY \\ DYSMENORRHEA: INTEGRATIVE REVIEW
}

Bianca Aparecida Brito da Silva ${ }^{1^{*}}$, Thamyris Alexandre Salles ${ }^{1}$, Glauteice Freitas Guedes $^{2}$.

\author{
${ }^{1}$ Universidade Federal de Alfenas, Alfenas, MG, Brasil. \\ ²Universidade Cruzeiro do Sul, São Paulo, SP, Brasil. \\ *biancabrito_1989@hotmail.com
}

Recebido em: 21/11/2019; Aceito em: 27/10/2020.

\section{RESUMO}

A dismenorreia primária é considerada um distúrbio álgico em região suprapúbica associada também a outros sintomas, que ocorrem no período menstrual, provocando redução da qualidade das atividades de vida diária, atenuação da produtividade laboral e absenteísmo, constituindo-se um problema de saúde pública, o que torna imprescindível a busca por tratamentos eficientes. Diante dessa problemática e do grande número de mulheres que sofrem e são prejudicadas por esse distúrbio a pergunta norteadora desta pesquisa foi: "Quais as terapias não farmacológicas que podem combater ou amenizar a dismenorreia?" O objetivo deste estudo foi identificar na literatura evidências científicas sobre terapias não farmacológicas que podem ser utilizadas para 0 alívio da dismenorreia primária. Trata-se de uma revisão integrativa de literatura, que explorou artigos nas bases de dados Literatura Latino-Americana e do Caribe em Ciências da Saúde e Medical Literature Analysis and Retrieval System Online. O corpus da revisão foi composto por 29 publicações. Os métodos não farmacológicos relevantes para a redução da dismenorreia primária encontrados foram: acupressão; acupuntura; aromaterapia; bandagem elástica funcional; estimulação elétrica nervosa transcutânea; exercícios físicos; massagem aromaterapêutica; massagem terapêutica; moxabustão; pilates; raios infravermelhos; termoterapia e yoga. Os estudos apontaram que estes métodos foram efetivos para o alívio da dismenorreia primária. Nesse âmbito, é importante que os profissionais de saúde, tenham conhecimento acerca da efetividade destas terapias, o que contribui para a integralidade do cuidado, orientação eficiente e assertiva quanto aos inúmeros métodos alternativos existentes para estes quadros dolorosos.

Palavras-chave: Dismenorreia. Enfermagem. Menstruação. Saúde da Mulher.

\section{ABSTRACT}

Primary dysmenorrhea is considered a pain disorder in the suprapubic region also associated with other symptoms, which occur in the menstrual period, causing a reduction in the quality of activities of daily living, attenuation of labor 
productivity and absenteeism, constituting a public health problem, which makes a search for efficient treatments essential. Faced with this problem and the large number of women who request and are harmed by this disorder, the guiding question of this research was: "What are the non-pharmacological therapies that can combat or ameliorate dysmenorrhea?" The aim of the study was to identify the scientific evidence on non-pharmacological therapies that can be used for the relief of primary dysmenorrhea. It is an integrative literature review, which explored articles in the Latin American and Caribbean Literature in Health Sciences and Medical Literature Analysis and Retrieval System Online databases. The review corpus consisted of 29 publications. The nonpharmacological methods relevant to the reduction of primary dysmenorrhea found were: acupressure; acupuncture; aromatherapy; functional elastic bandage; transcutaneous electrical nerve stimulation; physical exercises; aromatherapy massage; massage massage; moxibustion; pilates; infrared rays; thermotherapy and yoga. Studies have shown that these methods were effective in relieving primary dysmenorrhea. In this context, it is important that health professionals have knowledge about the effectiveness of these therapies, which contributes to the integrality of care, efficient and assertive guidance regarding the numerous alternative methods that exist for these painful conditions.

Keywords: Dysmenorrhea. Menstruation. Nursing. Women's Health.

\section{INTRODUÇÃO}

Dismenorreia é uma palavra derivada do termo grego: dys (difícil, dolorosa, anormal), meno (mês), rhoe (fluxo), que corresponde a um distúrbio ginecológico que provoca durante o período menstrual dor crônica e espasmódica na região suprapúbica (LUMSDEN; ILIODROMITI, 2015). Este desconforto se caracteriza por dor acentuada, em forma de cólica, manifestada em região abdominal inferior ou lombar que começam antes ou no início da menstruação, durando de um a três dias, provocando mensalmente limitações para inúmeras mulheres (ALVES et al., 2016; SANTOS et al., 2017).

Esse distúrbio pode ser classificado quanto à sua intensidade álgica como leve, moderada e grave, e no que concerne à etiologia, como primária - que ocorre sem que haja uma doença pélvica e secundária - na presença de doença pélvica (BARACAT; FONSECA; BAGNOLI, 2015). É uma das queixas ginecológicas mais frequentes dentre as mulheres de todo o mundo e afeta a qualidade de vida (LUMSDEN; ILIODROMITI, 2015).

Quando intensa, essa afecção está ligada à morbidade significante, podendo interferir no desempenho de atividades de vida diárias e levar ao absenteísmo escolar, do trabalho e de outros desempenhos, principalmente nos primeiros dias do ciclo menstrual (ALVES et al., 2016, SMITH; KAUNITZ, 2017).

À vista disso, torna-se imprescindível o tratamento deste distúrbio, podendo ser empregado três abordagens: a farmacológica, a cirúrgica e a não farmacológica. Os métodos não farmacológicos promovem 0 alívio do desconforto da dismenorreia (BAVIL et al., 2016), como o uso de terapias integrativas e complementares, massagem, terapia manual (PINHO et al., 2017), 
Pilates, atividade física, termoterapia, crioterapia, plantas, eletroestimulação nervosa transcutânea (BAVIL et al., 2016; SANTOS et al., 2017).

Para auxiliar a mulher com dismenorreia é importante que os profissionais de saúde, inclusive o enfermeiro, forneçam orientações acerca dos métodos não farmacológicos que propiciam o alívio do desconforto. Assim é fundamental que os profissionais conheçam os métodos existentes, para que as mulheres sejam orientadas de modo mais eficiente, possibilitando mais conforto nesse período (SANTOS et al., 2017).

O tratamento por meio de métodos não farmacológicos pode ser orientado ou aplicado em alguns casos pelo enfermeiro, caso possua capacitação, ele pode auxiliar a mulher fornecendo informações sobre a doença, métodos não farmacológicos que proporcionam o alívio da dor proporcionando uma assistência ampliada (SANTOS et al., 2017).

Apesar da alta prevalência de dismenorreia entre as mulheres jovens, constituindo-se em um problema de saúde pública, na literatura atual se nota uma carência de estudos acerca deste tema (NUNES et al., 2013; SANTOS et al., 2017).

Diante dessa problemática e pelo grande número de mulheres que sofrem e são prejudicadas por esse distúrbio, acredita-se ser um relevante problema de saúde pública. Assim, o presente estudo justifica-se pela necessidade da identificação de terapias não farmacológicas que possam ser utilizadas para amenizar a dismenorreia.

Assim, no intuito de contribuir com a disseminação dos métodos alternativos existentes, a melhoria do conhecimento científico dos profissionais de saúde e da práxis baseada em evidências, o presente estudo tem o objetivo de identificar na literatura evidências científicas sobre as terapias não farmacológicas que podem ser utilizadas para o alívio da dismenorreia primária.

\section{MATERIAL E MÉTODOS}

No presente estudo foi adotada a revisão integrativa da literatura como estratégia metodológica, que tem como objetivo sintetizar resultados verificados em estudos sobre uma temática de modo sistemático, ordenado e amplo. Ela proporciona 0 contato com informações mais abrangentes sobre um determinado assunto. Este tipo de estudo é composto por seis etapas (MENDES; SILVEIRA; GALVÃO, 2008), que serão demonstradas pelo presente estudo sobre as terapias não farmacológicas que podem combater ou amenizar a dismenorreia.

Fase 1 - Identificação do tema e seleção da hipótese ou questão da pesquisa. Nessa fase descreveu-se o propósito do estudo, que neste caso se trata de investigar quais as terapias não farmacológicas que podem combater ou amenizar a dismenorreia. Diante dessa problemática e do grande número de mulheres que sofrem e são prejudicadas por esse distúrbio, emerge a pergunta: "Quais as terapias não farmacológicas que podem combater ou amenizar a dismenorreia"? Essas terapias têm se mostrado efetivas?

Fase 2 - Estabelecimento de critérios para inclusão e exclusão de estudos/amostragem ou busca na literatura. Definiu-se como critérios de inclusão os artigos disponíveis na íntegra, publicados no período de janeiro de 
2014 a junho de 2018, nos idiomas português, espanhol e inglês e que respondessem a questão norteadora do estudo. Foram excluídos editoriais, cartas ao editor, resenhas, teses, dissertações, duplicados e aqueles artigos que não atendessem aos critérios de inclusão.

Nessa etapa foi realizada uma busca sistematizada nas bases de dados, com o intuito de explorar as publicações sobre o assunto. No estudo em questão, o processo de exploração teórica das terapias não farmacológicas que podem combater ou amenizar a dismenorreia, foi realizado nos meses de agosto e setembro de 2018, nas bases de dados: Literatura Latino-Americana e do Caribe em Ciências da Saúde (LILACS) e Medical Literature Analysis and Retrieval Sistem on-line (MEDLINE). Como estratégia de busca foram empregados os Descritores em Ciência da Saúde (DECS): dismenorreia, terapia, enfermagem, saúde da mulher e terapias complementares. A combinação dos descritores foi realizada com a utilização do operador booleano AND.

Fase 3 - Definição das informações a serem extraídas dos estudos selecionados/categorização dos estudos. Nesta fase definiu-se que seriam extraídas dos estudos as seguintes informações: título, autoria, ano de publicação, tipo de estudo, objetivo, conclusão e a classificação hierárquica quanto ao nível de evidência proposta por Melnyk, Fineout-Overholt (2019).

Fase 4 - Avaliação dos estudos incluídos. A seleção dos artigos encontrase descrita na Figura 1, apresentada nos resultados e discussão. Concernente ao nível de evidência dos estudos utilizou-se a seguinte classificação: nível I Revisões sistemáticas ou metanálise de Estudo Clínico com randomização, nível II - Estudo Clínico com randomização, nível III - Estudo Clínico sem randomização, nível IV - Coorte e Caso controle bem delineados, nível V Revisão sistemática de estudos descritivos/qualitativos, nível VI - Estudos oriundos de estudo descritivo/qualitativo e nível VII - oriundas de opinião de autoridades e/ou relatórios de comitês de especialistas (MELNYK, FINEOUTOVERHOLT, 2019).

Fase 5 - Interpretação dos resultados. Os dados foram organizados em quadros elaborados pelas autoras, que permitem explorar quais as terapias não farmacológicas que podem combater ou amenizar a dismenorreia e responder a questão norteadora desta revisão, sendo discutidos posteriormente.

Fase 6 - Apresentação da revisão/síntese do conhecimento. Os resultados alcançados por meio dos artigos que compõem essa revisão foram apresentados, sintetizados, discutidos e encontram-se durante a exposição dos resultados e discussão.

\section{RESULTADOS E DISCUSSÃO}

A busca nas bases de dados identificou 232 estudos, desses excluiu-se 200 por não abordarem a temática, três por duplicidade e oito por não serem artigos primários, sendo a amostra composta por 29 (100\%) artigos. Para melhor visualização organizou-se o fluxograma (Figura 1).

Os artigos incluídos foram caracterizados quanto ao código correspondente, autores, ano publicação e título (Quadro 1). Para melhor identificação dos artigos que compõe este estudo, organizou-se uma exposição em sequência alfanumérica iniciando em A1 até A29. 
Figura 1 - Fluxograma de seleção dos artigos que compõem a revisão integrativa de literatura, realizado no período de agosto e setembro de 2018

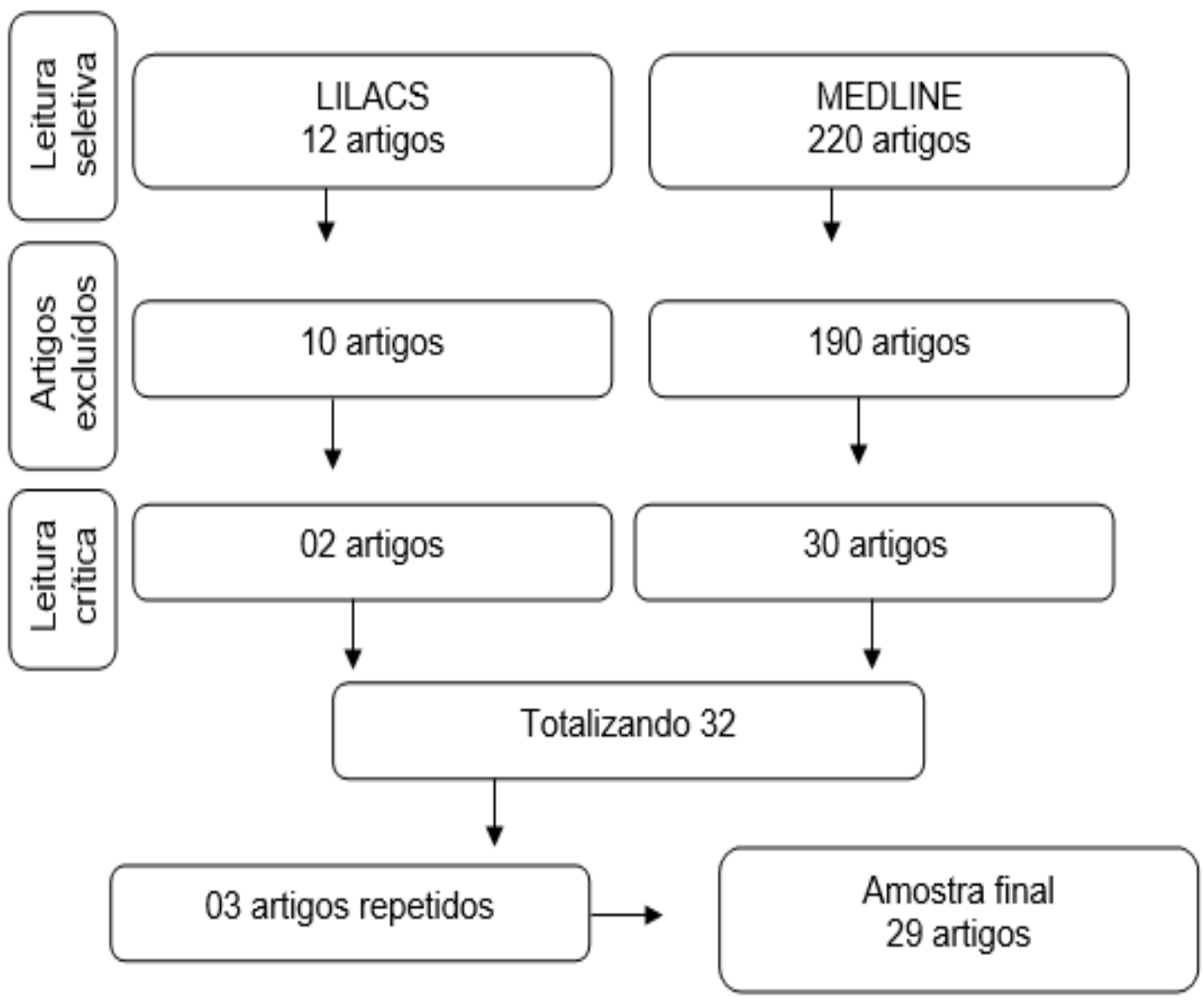

Fonte: os autores.

Quadro 1 - Caracterização das publicações incluídas no estudo quanto ao código, autores / ano e título do artigo

\begin{tabular}{|l|c|l|}
\hline Cód. & Autores / ano & \multicolumn{1}{c|}{ Título do artigo } \\
\hline A1 & $\begin{array}{c}\text { Abaraogu, Igwe e } \\
\text { Tabansi-ochiogu } \\
(2016)\end{array}$ & $\begin{array}{l}\text { Effectiveness of SP6 (Sanyinjiao) acupressure for relief of primary } \\
\text { dysmenorrhea symptoms: A systematic review with meta- and sensitivity } \\
\text { analyses. }\end{array}$ \\
\hline A2 & $\begin{array}{c}\text { Chen et al. } \\
(2015)\end{array}$ & $\begin{array}{l}\text { Effects of acupressure on menstrual distress and low back pain in } \\
\text { dysmenorrheic young adult women: an experimental study. }\end{array}$ \\
\hline A3 & $\begin{array}{c}\text { Cha e Sok (2016) } \\
\text { Effects of Auricular Acupressure Therapy on Primary Dysmenorrhea for } \\
\text { Female High School Students in South Korea. }\end{array}$ \\
\hline A4 & $\begin{array}{c}\text { Abaraogu e } \\
\text { Tabansi-ochuogu } \\
(2015)\end{array}$ & $\begin{array}{l}\text { As Acupressure Decreases Pain, Acupuncture May Improve Some Aspects } \\
\text { of Quality of Life for Women with Primary Dysmenorrhea: A Systematic } \\
\text { Review with Meta-Analysis. }\end{array}$ \\
\hline A5 & Woo et al. (2018) & $\begin{array}{l}\text { The efficacy and safety of acupuncture in women with primary } \\
\text { dysmenorrhea: A systematic review and meta-analysis. }\end{array}$ \\
\hline A6 & Xu et al. (2017) & $\begin{array}{l}\text { Effects of acupoint-stimulation for the treatment of primary dysmenorrhoea } \\
\text { compared with NSAIDs: a systematic review and meta-analysis of 19 } \\
\text { RCTs. }\end{array}$ \\
\hline
\end{tabular}


Quadro 1, cont.

\begin{tabular}{|c|c|c|}
\hline A7 & $\begin{array}{l}\text { Armour et al. } \\
\quad(2017)\end{array}$ & $\begin{array}{l}\text { The role of treatment timing and mode of stimulation in the treatment of } \\
\text { primary dysmenorrhea with acupuncture: An exploratory randomised } \\
\text { controlled trial. }\end{array}$ \\
\hline A8 & $\begin{array}{l}\text { Armour e Smith } \\
(2016)\end{array}$ & $\begin{array}{l}\text { Treating primary dysmenorrhoea with acupuncture: a narrative review of } \\
\text { the relationship between acupuncture 'dose' and menstrual pain outcomes. }\end{array}$ \\
\hline A9 & $\begin{array}{l}\text { Smith et al. } \\
(2016)\end{array}$ & Acupuncture for dysmenorrhoea. \\
\hline A10 & Liu et al. (2014) & $\begin{array}{l}\text { A randomized controlled trial of single point acupuncture in primary } \\
\text { dysmenorrheal }\end{array}$ \\
\hline A11 & Gao et al. (2015) & $\begin{array}{l}\text { The effect of moxibustion on alleviating menstrual pain in a population of } \\
\text { young nursing students: A prospective randomized cross-over pilot study }\end{array}$ \\
\hline A12 & $\begin{array}{l}\text { Yang et al. } \\
\text { (2015) }\end{array}$ & $\begin{array}{l}\text { Use of moxibustion to treat primary dysmenorrhea at two interventional } \\
\text { times: study protocol for a randomized controlled trial }\end{array}$ \\
\hline A13 & $\begin{array}{l}\text { Uysal et al. } \\
(2016)\end{array}$ & $\begin{array}{l}\text { Investigating the effect of rose essential oil in patients with primary } \\
\text { dysmenorrheal }\end{array}$ \\
\hline A14 & $\begin{array}{l}\text { Dehkordi, } \\
\text { Baharanchi e } \\
\text { Bekhradi (2014) }\end{array}$ & $\begin{array}{l}\text { Effect of lavender inhalation on the symptoms of primary dysmenorrhea } \\
\text { and the amount of menstrual bleeding: A randomized clinical trial }\end{array}$ \\
\hline A15 & $\begin{array}{l}\text { Sut e kahyaoglu- } \\
\text { sut (2017) }\end{array}$ & $\begin{array}{l}\text { Effect of aromatherapy massage on pain in primary dysmenorrhea: A meta- } \\
\text { analysis }\end{array}$ \\
\hline A16 & $\begin{array}{l}\text { Shahr et al. } \\
(2015)\end{array}$ & $\begin{array}{l}\text { The effect of self-aromatherapy massage of the abdomen on the primary } \\
\text { dysmenorrhea }\end{array}$ \\
\hline A17 & $\begin{array}{c}\text { Azima et al. } \\
(2015)\end{array}$ & $\begin{array}{l}\text { Comparison of the Effect of Massage Therapy and Isometric Exercises on } \\
\text { Primary Dysmenorrhea: A Randomized Controlled Clinical Trial }\end{array}$ \\
\hline A18 & $\begin{array}{l}\text { Stallbaum et al. } \\
(2016)\end{array}$ & $\begin{array}{l}\text { Efeitos da bandagem funcional elástica sobre a dismenorreia primaria em } \\
\text { universitárias }\end{array}$ \\
\hline A19 & $\begin{array}{l}\text { Brown e Brown } \\
\text { (2017) }\end{array}$ & WITHDRAWN: Exercise for dysmenorrhoea \\
\hline A20 & $\begin{array}{l}\text { Kannan et al. } \\
(2015 b)\end{array}$ & $\begin{array}{l}\text { Menstrual pain and quality of life in women with primary dysmenorrhea: } \\
\text { Rationale, design, and interventions of a randomized controlled trial of } \\
\text { effects of a treadmill-based exercise intervention }\end{array}$ \\
\hline A21 & $\begin{array}{l}\text { Kannan et al. } \\
(2015 a)\end{array}$ & $\begin{array}{l}\text { Vigorous exercises in the management of primary dysmenorrhea: a } \\
\text { feasibility study. }\end{array}$ \\
\hline A22 & $\begin{array}{l}\text { Fonseca et al. } \\
\quad(2016)\end{array}$ & $\begin{array}{l}\text { A influência do método Pilates na flexibilidade muscular, sintomas e } \\
\text { qualidade de vida em mulheres com dismenorreia primária }\end{array}$ \\
\hline A23 & Tsai (2016) & $\begin{array}{l}\text { Effect of Yoga Exercise on Premenstrual Symptoms among Female } \\
\text { Employees in Taiwan }\end{array}$ \\
\hline A24 & $\begin{array}{l}\text { Yang e Kim } \\
(2016)\end{array}$ & $\begin{array}{l}\text { Effects of a Yoga Program on Menstrual Cramps and Menstrual Distress in } \\
\text { Undergraduate Students with Primary Dysmenorrhea: A Single-Blind, } \\
\text { Randomized Controlled Trial }\end{array}$ \\
\hline A25 & $\begin{array}{l}\text { Lauretti et al. } \\
\qquad(2015)\end{array}$ & $\begin{array}{l}\text { The New Portable Transcutaneous Electrical Nerve Stimulation Device } \\
\text { Was Efficacious in the Control of Primary Dysmenorrhea Cramp Pain }\end{array}$ \\
\hline A26 & $\begin{array}{l}\text { Bai, Bai e Yang } \\
(2017)\end{array}$ & $\begin{array}{l}\text { Effect of transcutaneous electrical nerve stimulation therapy for the } \\
\text { treatment of primary dysmenorrheal }\end{array}$ \\
\hline A27 & $\begin{array}{l}\text { Igwea, Tabansi- } \\
\text { ochuogu e } \\
\text { Abaraogu (2016) }\end{array}$ & $\begin{array}{l}\text { TENS and heat therapy for pain relief and quality of life improvement in } \\
\text { individuals with primary dysmenorrhea: A systematic review }\end{array}$ \\
\hline A28 & Lee et al. (2015) & $\begin{array}{l}\text { Efficacy of the device combining high-frequency transcutaneous electrical } \\
\text { nerve stimulation and thermotherapy for relieving primary dysmenorrhea: a } \\
\text { randomized, single-blind, placebo-controlled trial }\end{array}$ \\
\hline A29 & Lee e KIM (2017) & red rays on female menstrual pain in Korea \\
\hline
\end{tabular}

Fonte: os autores.

A síntese dos estudos, com relação ao tipo de estudo, objetivos, terapia utilizada e conclusão é apresentada no Quadro 2. 
Quadro 2 - Apresentação do tipo de estudo, objetivo, terapia utilizada, conclusão e nível de evidência dos artigos

\begin{tabular}{|c|c|c|c|}
\hline Cód. & $\begin{array}{l}\text { Tipo de } \\
\text { estudo }\end{array}$ & Objetivo & Conclusão \\
\hline $\mathrm{A} 1$ & $\begin{array}{l}\text { Revisão } \\
\text { sistemática }\end{array}$ & $\begin{array}{l}\text { Revisar as evidências disponíveis } \\
\text { para o alívio dos sintomas da } \\
\text { dismenorreia primária com uso da } \\
\text { acupressão SP6 (Sanyinjiao), bem } \\
\text { como as experiências dos pacientes } \\
\text { com esta intervenção. }\end{array}$ & $\begin{array}{l}\text { A acupressão mostrou-se eficaz } \\
\text { quando aplicada por profissional } \\
\text { treinado para alguns sintomas de } \\
\text { dismenorreia primária. Os resultados } \\
\text { sugerem que a acupressão } \\
\text { autoadministrada pode ser promissora } \\
\text { para o alívio dos sintomas. }\end{array}$ \\
\hline $\mathrm{A} 2$ & $\begin{array}{l}\text { Estudo } \\
\text { experimental }\end{array}$ & $\begin{array}{l}\text { Examinar os efeitos da acupressão } \\
\text { no desconforto menstrual e } \\
\text { lombalgia em mulheres com } \\
\text { dismenorreia. }\end{array}$ & $\begin{array}{l}\text { O estudo demonstrou efeitos de curto, } \\
\text { médio e longo prazo da acupressão } \\
\text { sobre o alívio do desconforto } \\
\text { menstrual e confirmou que pode ser } \\
\text { usada como um tratamento não } \\
\text { farmacológico seguro e fácil. }\end{array}$ \\
\hline A3 & $\begin{array}{l}\text { Ensaio clínico } \\
\text { randomizado }\end{array}$ & $\begin{array}{l}\text { Examinar o efeito da terapia de } \\
\text { acupressão } \\
\text { dismenorreia auricular } \\
\text { estudantes do ensina ma nédio na } \\
\text { Coreia do Sul. }\end{array}$ & $\begin{array}{l}\text { A terapia foi eficaz para o alívio dos } \\
\text { sintomas da dismenorreia primária em } \\
\text { estudantes do ensino médio na Coreia } \\
\text { do Sul. Para sua viabilidade é } \\
\text { necessário treinar e aprender as } \\
\text { posições exatas dos pontos de } \\
\text { acupuntura no ouvido. }\end{array}$ \\
\hline A4 & $\begin{array}{l}\text { Revisão } \\
\text { sistemática } \\
\text { com meta- } \\
\text { análise }\end{array}$ & $\begin{array}{l}\text { Obter evidências sobre a eficácia da } \\
\text { acupuntura e intervenções de } \\
\text { acupressão. }\end{array}$ & $\begin{array}{l}\text { A acupressão mostrou evidências de } \\
\text { alívio da dor, enquanto a acupuntura } \\
\text { melhorou os componentes mentais e } \\
\text { físicos da qualidade de vida. }\end{array}$ \\
\hline A5 & $\begin{array}{l}\text { Revisão } \\
\text { sistemática } \\
\text { com meta- } \\
\text { análise }\end{array}$ & $\begin{array}{l}\text { Avaliar as evidências sobre a } \\
\text { eficácia e segurança da acupuntura } \\
\text { na dismenorreia primária. }\end{array}$ & $\begin{array}{l}\text { A acupuntura pode ser usada como } \\
\text { um tratamento eficaz e seguro para } \\
\text { mulheres com dismenorreia primária. }\end{array}$ \\
\hline A6 & $\begin{array}{l}\text { Revisão } \\
\text { sistemática } \\
\text { com meta- } \\
\text { análise }\end{array}$ & $\begin{array}{lccc}\text { Determinar } & \text { a } & \text { eficácia } & \text { da } \\
\text { estimulação } & \text { de } & \text { pontos } & \text { de } \\
\text { acupuntura em comparação com os } \\
\text { anti-inflamatórios não esteroidais no } \\
\text { tratamento da } & \text { dismenorreia } \\
\text { primária. } & & \end{array}$ & $\begin{array}{l}\text { A estimulação por pontos de } \\
\text { acupuntura pode aliviar a dor de forma } \\
\text { eficaz e oferece vantagens no } \\
\text { aumento da eficácia geral. }\end{array}$ \\
\hline A7 & $\begin{array}{l}\text { Ensaio clínico } \\
\text { randomizado }\end{array}$ & $\begin{array}{l}\text { Examinar o efeito do tempo de } \\
\text { tratamento no uso de } \\
\text { eletroacupuntura manual sobre os } \\
\text { sintomas da dismenorreia primária. }\end{array}$ & $\begin{array}{l}\text { O tratamento com acupuntura reduziu } \\
\text { a intensidade e a duração da dor } \\
\text { menstrual após três meses de } \\
\text { tratamento e foi mantida por até um } \\
\text { ano. O efeito da mudança do modo de } \\
\text { estimulação ou frequência do } \\
\text { tratamento na dor menstrual não foi } \\
\text { significativo. }\end{array}$ \\
\hline A8 & $\begin{array}{l}\text { Revisão } \\
\text { narrativa }\end{array}$ & $\begin{array}{l}\text { Explorar a relação entre a 'dose' da } \\
\text { intervenção de acupuntura e os } \\
\text { resultados da dor menstrual. }\end{array}$ & $\begin{array}{l}\text { Os resultados apontaram relação } \\
\text { entre o tempo de tratamento e o modo } \\
\text { de estimulação com agulha na } \\
\text { redução da intensidade da dor } \\
\text { menstrual. }\end{array}$ \\
\hline A9 & $\begin{array}{l}\text { Revisão } \\
\text { sistemática }\end{array}$ & $\begin{array}{l}\text { Determinar a eficácia e segurança } \\
\text { da acupuntura e acupressão no } \\
\text { tratamento da dismenorreia primária } \\
\text { quando comparada com um } \\
\text { placebo, nenhum tratamento ou } \\
\text { tratamento médico convencional. }\end{array}$ & $\begin{array}{l}\text { Não houve evidências suficientes para } \\
\text { demonstrar se a acupuntura ou } \\
\text { acupressão são ou não eficazes no } \\
\text { tratamento da dismenorreia primária. }\end{array}$ \\
\hline
\end{tabular}


Quadro 2, cont.

\begin{tabular}{|c|c|c|c|}
\hline A10 & $\begin{array}{l}\text { Ensaio clínico } \\
\text { controlado e } \\
\text { randomizado }\end{array}$ & $\begin{array}{lccc}\text { Avaliar o } & \text { efeito imediato } & \text { da } \\
\text { acupuntura } & \text { em pontos } & \text { de } \\
\text { acupuntura } & \text { específicos } & \text { para } \\
\text { dismenorreia } & \text { primária, } & \text { em } \\
\text { comparação com pontos } & \text { de } \\
\text { acupuntura e de não-acupuntura. }\end{array}$ & $\begin{array}{l}\text { A acupuntura em pontos específicos } \\
\text { de acupuntura produziu um efeito } \\
\text { estatisticamente, mas } \\
\text { clinicamente, significativo, em } \\
\text { comparação com pontos de } \\
\text { acupuntura e de não-acupuntura em } \\
\text { pacientes com dismenorreia primária. }\end{array}$ \\
\hline A11 & $\begin{array}{l}\text { Ensaio clínico } \\
\text { randomizado }\end{array}$ & $\begin{array}{l}\text { Investigar o efeito da moxabustão no } \\
\text { alívio dos sintomas da dismenorreia } \\
\text { em jovens estudantes de } \\
\text { enfermagem na China. }\end{array}$ & $\begin{array}{l}\text { Os resultados sugeriram que a terapia } \\
\text { foi eficaz para aliviar a dor menstrual e } \\
\text { os sintomas da dismenorreia primária. }\end{array}$ \\
\hline A12 & $\begin{array}{l}\text { Protocolo de } \\
\text { estudo para } \\
\text { um ensaio } \\
\text { clínico } \\
\text { randomizado }\end{array}$ & $\begin{array}{l}\text { Avaliar a eficácia e segurança da } \\
\text { moxabustão para dismenorreia } \\
\text { primária,e determinar se o momento } \\
\text { ideal de tratamento para é antes ou } \\
\text { depois do início da dor menstrual. }\end{array}$ & $\begin{array}{l}\text { Acredita-se que este ensaio fornecerá } \\
\text { boas evidências } \\
\text { para demonstrar a eficácia e } \\
\text { segurança da moxabustão } \\
\text { para pacientes com dismenorreia } \\
\text { primária. }\end{array}$ \\
\hline A13 & $\begin{array}{l}\text { Estudo } \\
\text { experimental }\end{array}$ & $\begin{array}{l}\text { Avaliar os efeitos do óleo essencial } \\
\text { de rosa na dismenorreia primária. }\end{array}$ & $\begin{array}{l}\text { Concluiu-se que a aromaterapia com } \\
\text { óleo essencial de rosa, um adjuvante } \\
\text { aos métodos convencionais de } \\
\text { tratamento e pode ser benéfica para o } \\
\text { alívio da dismenorreia primária. }\end{array}$ \\
\hline A14 & $\begin{array}{l}\text { Ensaio clínico } \\
\text { randomizado }\end{array}$ & $\begin{array}{l}\text { Explorar o efeito da inalação de } \\
\text { lavanda nos sintomas da } \\
\text { dismenorreia primária e na } \\
\text { quantidade de sangramento } \\
\text { menstrual em estudantes. }\end{array}$ & $\begin{array}{l}\text { Este estudo demonstrou eficácia no } \\
\text { alívio dos sintomas, sugerindo que } \\
\text { pode ser aplicada de forma segura. }\end{array}$ \\
\hline A15 & $\begin{array}{l}\text { Revisão } \\
\text { sistemática } \\
\text { com meta- } \\
\text { análise }\end{array}$ & $\begin{array}{l}\text { Investigar o efeito da massagem de } \\
\text { aromaterapia na dismenorreia } \\
\text { primária }\end{array}$ & $\begin{array}{l}\text { A massagem de aromaterapia } \\
\text { abdominal com óleos essenciais é um } \\
\text { método complementar eficaz para o } \\
\text { alívio da dismenorreia primária. }\end{array}$ \\
\hline A16 & $\begin{array}{l}\text { Ensaio clínico } \\
\text { randomizado }\end{array}$ & $\begin{array}{l}\text { Avaliar o efeito da massagem } \\
\text { terapêutica junto com } \\
\text { óleo de rosa para dismenorreia } \\
\text { primária. }\end{array}$ & $\begin{array}{l}\text { A massagem com aromaterapia reduz } \\
\text { a dismenorreia primária, em } \\
\text { comparação com a massagem } \\
\text { terapêutica isolada. }\end{array}$ \\
\hline A17 & $\begin{array}{l}\text { Ensaio clínico } \\
\text { controlado e } \\
\text { randomizado }\end{array}$ & $\begin{array}{l}\text { Comparar os efeitos da massagem e } \\
\text { exercícios isométricos na } \\
\text { dismenorreia primária. }\end{array}$ & $\begin{array}{l}\text { A massagem terapêutica e os } \\
\text { exercícios isométricos foram eficazes } \\
\text { na redução de alguns sintomas da } \\
\text { dismenorreia primária. }\end{array}$ \\
\hline A18 & $\begin{array}{l}\text { Ensaio clínico } \\
\text { randomizado }\end{array}$ & $\begin{array}{l}\text { Avaliar os efeitos da bandagem } \\
\text { funcional sobre a dor e asatividades } \\
\text { de vida diária de estudantes } \\
\text { universitárias com dismenorreia } \\
\text { primária. }\end{array}$ & $\begin{array}{l}\text { A bandagem demonstrou-se benéfica } \\
\text { na dismenorreia primária, com efeitos } \\
\text { positivos sobre a redução da } \\
\text { intensidade e a duração da dor. }\end{array}$ \\
\hline A19 & $\begin{array}{l}\text { Revisão } \\
\text { sistemática }\end{array}$ & $\begin{array}{l}\text { Avaliar as evidências da eficácia do } \\
\text { exercício no tratamento da } \\
\text { dismenorreia. }\end{array}$ & $\begin{array}{l}\text { Os autores concluíram que os } \\
\text { resultados desta revisão são limitados } \\
\text { a um único ensaio randomizado de } \\
\text { qualidade e mais pesquisas são } \\
\text { necessárias. }\end{array}$ \\
\hline A20 & $\begin{array}{l}\text { Ensaio clínico } \\
\text { controlado e } \\
\text { randomizado }\end{array}$ & $\begin{array}{l}\text { Avaliar a eficácia dos exercícios } \\
\text { físicos para reduzir a intensidade da } \\
\text { dor e melhorar a qualidade de vida } \\
\text { em mulheres com dismenorreia } \\
\text { primária. }\end{array}$ & $\begin{array}{l}\text { O exercício é uma intervenção de } \\
\text { baixo custo que pode ser ensinada e } \\
\text { adaptada a um indivíduo. Se o } \\
\text { benefício for comprovado, ele pode } \\
\text { ser continuado } \\
\text { de forma independente e indefinida } \\
\text { para controle da dismenorreia } \\
\text { primária. }\end{array}$ \\
\hline
\end{tabular}


Quadro 2, cont.

\begin{tabular}{|c|c|c|c|}
\hline A21 & $\begin{array}{l}\text { Estudo quase } \\
\text { experimental }\end{array}$ & $\begin{array}{l}\text { Examinar a viabilidade de uma } \\
\text { intervenção com exercícios para } \\
\text { reduzir a dismenorreia primária e } \\
\text { obter resultados preliminares para } \\
\text { estimar o tamanho da amostra para } \\
\text { um futuro ensaio clínico } \\
\text { randomizado. }\end{array}$ & $\begin{array}{l}\text { Os resultados deste estudo } \\
\text { demostraram que os exercícios } \\
\text { podem ser eficazes na redução da dor } \\
\text { associada à dismenorreia primária. }\end{array}$ \\
\hline A22 & $\begin{array}{l}\text { Ensaio clinico } \\
\text { não controlado }\end{array}$ & $\begin{array}{l}\text { Investigar a influência dos exercícios } \\
\text { do método Pilates sobre a } \\
\text { flexibilidade muscular, } \\
\text { sintomatologia e qualidade de vida } \\
\text { em mulheres com dismenorreia } \\
\text { primária. }\end{array}$ & $\begin{array}{l}\text { Os exercícios do método Pilates } \\
\text { proporcionaram melhora da } \\
\text { flexibilidade muscular, redução da dor } \\
\text { e dos desconfortos, indicando ser } \\
\text { alternativa eficaz para o tratamento } \\
\text { sintomático da dismenorreia primária. }\end{array}$ \\
\hline A23 & $\begin{array}{l}\text { Estudo quase } \\
\text { experimental }\end{array}$ & $\begin{array}{l}\text { Avaliar os feitos de exercícios de } \\
\text { ioga por } 12 \text { semanas nos sintomas } \\
\text { pré-menstruais em mulheres } \\
\text { menstruadas em Taiwan. }\end{array}$ & $\begin{array}{l}\text { Concluiu-se que as mulheres que } \\
\text { participaram da intervenção relataram } \\
\text { menos sintomas pré-menstruais } \\
\text { associados a um menor risco de dor } \\
\text { menstrual. }\end{array}$ \\
\hline A24 & $\begin{array}{l}\text { Ensaio clínico } \\
\text { randomizado }\end{array}$ & $\begin{array}{l}\text { Investigar os efeitos da ioga sobre } \\
\text { cólicas menstruais e desconforto } \\
\text { menstrual em estudantes de } \\
\text { graduação com dismenorreia } \\
\text { primária. }\end{array}$ & $\begin{array}{l}\text { Conclui-se que as intervenções de } \\
\text { ioga podem reduzir as cólicas } \\
\text { menstruais e o desconforto menstrual } \\
\text { em estudantes com dismenorréia } \\
\text { primária. }\end{array}$ \\
\hline A25 & $\begin{array}{l}\text { Ensaio clínico } \\
\text { randomizado }\end{array}$ & $\begin{array}{lrr}\text { Avaliar a eficácia e segurança de um } \\
\text { novo dispositivo portátil } & \text { TENS } \\
\text { (Estimulaçao } & \text { Elétrica } & \text { Nervosa } \\
\text { Transcutânea) } & \text { para } & \text { cólicas } \\
\text { menstruais. } & & \\
\text { mens. }\end{array}$ & $\begin{array}{l}\text { O dispositivo TENS induziu o início } \\
\text { imediato do alívio da dor e melhorou a } \\
\text { qualidade de vida, sem efeitos } \\
\text { adversos, em pacientes com dores } \\
\text { associadas à dismenorreia. }\end{array}$ \\
\hline A26 & $\begin{array}{l}\text { Ensaio clínico } \\
\text { randomizado }\end{array}$ & $\begin{array}{l}\text { Investigar o efeito e a segurança da } \\
\text { terapia de TENS para o alívio da dor } \\
\text { em mulheres com dismenorreia } \\
\text { primária. }\end{array}$ & $\begin{array}{l}\text { A TENS foi eficaz e segura no alívio da } \\
\text { dor em participantes com } \\
\text { dismenorreia primária. }\end{array}$ \\
\hline A27 & $\begin{array}{l}\text { Revisão } \\
\text { sistemática }\end{array}$ & $\begin{array}{l}\text { Sintetizar evidências para a eficácia } \\
\text { da TENS e intervenções de terapia } \\
\text { térmica de ensaios clínicos } \\
\text { randomizados. }\end{array}$ & $\begin{array}{l}\text { A terapia TENS e térmica mostraram- } \\
\text { se como potenciais coadjuvantes no } \\
\text { tratamento da dismenorreia primária. }\end{array}$ \\
\hline A28 & $\begin{array}{l}\text { Ensaio clínico } \\
\text { randomizado }\end{array}$ & $\begin{array}{l}\text { Investigar a eficácia e segurança da } \\
\text { terapia combinada com TENS de } \\
\text { alta frequência e termoterapia no } \\
\text { alívio da dismenorreia primária. }\end{array}$ & $\begin{array}{l}\text { A combinação de TENS e } \\
\text { termoterapia foram eficazes no alívio } \\
\text { da dor aguda em mulheres com } \\
\text { dismenorreia primária. }\end{array}$ \\
\hline A29 & $\begin{array}{l}\text { Ensaio clínico } \\
\text { randomizado }\end{array}$ & $\begin{array}{l}\text { Avaliar os efeitos dos raios } \\
\text { infravermelhos na dismenorreia } \\
\text { primária em mulheres coreanas. }\end{array}$ & $\begin{array}{l}\text { Os raios infravermelhos próximos ao } \\
\text { corpo podem ser usados para aliviar a } \\
\text { dor menstrual e melhorar a qualidade } \\
\text { de vida de mulheres com dismenorreia } \\
\text { na Coreia. }\end{array}$ \\
\hline
\end{tabular}

Fonte: os autores.

Quanto à classificação hierárquica do nível de evidência dos estudos que compuseram a amostra, pode-se observar que 13 (45\%) (A2, A3, A7, A10, A11, A14, A16, A20, A24, A25, A26, A28, A29) apresentaram evidência nível II, 10 (35\%) (A1, A4, A5, A6, A9, A15, A17, A18, A19, A27) com nível I, quatro estudos (14\%) (A13, A21, A22, A23) apresentaram nível III, um (3\%) (A8) nível IV e um (3\%) (A12) nível VI.

Com a realização desta revisão considera-se que a maioria dos artigos analisados foram bem delineados, com alta qualidade metodológica e apresentaram evidências fortes (nível I e II). Essa avaliação tem como objetivos determinar a confiança no uso dos resultados, o que possibilita subsidiar a 
tomada de decisões clínicas e fortalecer o conhecimento atual da temática (MELNYK et al., 2019).

Conforme os estudos analisados nesta pesquisa, observaram-se treze distintos métodos não farmacológicos que podem ser utilizados para o alívio de quadros dolorosos do período menstrual. Constatou-se que seis (20,69\%) (A5, A6, A7, A8, A9, A10) abordavam a acupuntura; três (10,34\%) (A1, A2, A3) acupressão; três (10,34\%) (A19, A20, A21) exercícios físicos; duas (6,9\%) (A13; A14) aromaterapia; duas (6,9\%) (A25; A26) estimulação elétrica nervosa transcutânea (TENS); duas (6,9\%) (A27; A28) TENS associada à termoterapia; duas $(6,9 \%)$ (A15, A16) massagem aromaterapêutica; duas $(6,9 \%)(A 11, A 12)$ moxabustão; duas (6,9\%) (A23, A24) loga; um (3,45\%) (A4) acupuntura associada à acupressão; um (3,45\%) (A18) bandagem elástica funcional; um $(3,45 \%)$ (A17) massagem terapêutica; um (3,45\%) (A22) Pilates e um $(3,45 \%)$ (A29) raios infravermelhos.

Estudo realizado por Santos et al. (2017) com o intuito de reunir terapias não farmacológicas para amenizar a dismenorreia, constatou significativa redução dos sintomas álgicos causados pela dismenorreia por meio de métodos como o exercício físico, Pilates, crioterapia, termoterapia, TENS, massagem e chá de ervas.

A seguir apresentam-se as terapias não farmacológicas estudadas.

\section{Acupuntura, Acupressão e Moxabustão}

A acupuntura é uma técnica baseada na Medicina Tradicional Chinesa que busca o alívio e a cura de doenças por meio de aplicação de agulhas e moxas estimulando os "Pontos de Acupuntura", assim reestabelecendo o equilíbrio através da liberação de neurotransmissores e hormônios responsáveis pela harmonia do organismo (DIAS; MELO, 2014).

Os estudos A1, A5, A6, e A7 constataram que as sessões deste procedimento colaboraram para o impacto positivo na qualidade de vida, resultando em sensação de bem-estar evidenciando resolutividade no tratamento da dor menstrual, as pesquisas apontam a acupuntura como um tratamento eficaz e seguro tanto de forma isolada como coadjuvante ao tratamento convencional da dismenorreia primária.

Os autores dos estudos A8, A9 e A10 sugerem haver relação entre redução da dor com uso de acupuntura, porém mais estudos são necessários.

A acupressão busca o equilíbrio energético do ser humano com ele mesmo e com o espaço, ela parte dos mesmos princípios da acupuntura, por meio do estímulo em pontos do corpo, envolve a pressão com um dos dedos com intensidade média ou leve, pode manter-se firme ou circundar a pele em pontos específicos, a ser realizada por profissionais de saúde e/ou pela própria pessoa, contribuindo para o bem estar e melhora da qualidade de vida (URICH, 1980; PIVETA, 2015).

O uso da terapia de acupressão auricular foi uma intervenção eficaz para o alívio da dismenorreia primária no estudo A3. Na pesquisa A2 o grupo experimental teve significativamente menor sofrimento menstrual após a aplicação da terapia e no estudo A1 os resultados se mostraram promissores para o alívio álgico, porém mais pesquisas são necessárias. 
A moxabustão é um método terapêutico que tem o objetivo de usar substâncias ou ervas para aquecer pontos de acupuntura ou áreas do corpo. A matéria prima mais utilizada para a combustão é a folha da planta Artemísia vulgaris, que possui propriedade antiinflamatória, cicatrizante, dispersa o frio e a umidade, regula a circulação e a energia. Várias técnicas podem ser utilizadas, como cones acesos colocados diretamente sobre os pontos ou áreas selecionadas, bastões de moxa de vários tamanhos que são posicionados sobre a região a ser tratada, sem tocá-la, dentre outros (YAMAMURA, 2001; VILLAHERMOSA et al., 2013).

Segundo os resultados do estudo A11, a moxabustão foi eficaz para aliviar a dor menstrual e sintomas de jovens universitárias na China, esta terapia pode ser recomendada como uma intervenção não-farmacológica, apresenta bom custo-benefício, prática e segura na autoadministração em casa. No estudo A12, os autores constataram que a terapia de moxabustão foi eficaz para aliviar a dor menstrual e sintomas da população feminina avaliada em seu estudo.

\section{Aromaterapia, Massagem Aromaterapêutica e Massagem Terapêutica}

Aromaterapia é considerada uma Terapia Complementar de saúde que utiliza concentrados voláteis extraídos de plantas também chamados óleos essenciais, com a finalidade de melhorar o bem-estar físico, mental e emocional (GNATTA et al., 2016). Os aromas colocam o indivíduo em contato com seu íntimo e com a natureza, preparando para o sono, repouso, estado de alerta, criatividade, dentre outros. O uso da massagem terapêutica integra um conjunto de práticas com manobras que objetivam favorecer a saúde e o equilíbrio com o corpo, proporcionando efeitos fisiológicos, psicológicos, na dor, dentre outros, reduzindo os malefícios físicos e emocionais (LIMA, 2016).

As pesquisas citadas nesta revisão revelaram que o uso de aromaterapia e massagem aromaterapêutica podem ser benéficos e eficazes para o alívio das dores menstruais (A13, A14, A15, A16) e o uso da massagem terapêutica também associada com os exercícios isométricos se demonstraram eficazes, reduzindo alguns sintomas da dismenorreia (A17).

\section{Bandagem Elástica Funcional}

A Bandagem elástica funcional traduz-se em uma técnica que pode auxiliar na melhora da integração sensorial do corpo com o ambiente, fornecendo a propriocepção local e consequente melhora no equilíbrio postural estático e dinâmico (CAMPOS et al., 2016).

Segundo estudo A18, a Bandagem Elástica Funcional quando aplicada de forma correta, é eficaz na redução da intensidade e duração da dismenorreia primária, trata-se de uma proposta de tratamento não invasiva e com mínimos efeitos colaterais, prevenindo a interferência desta condição sobre as atividades de vida diárias e também sobre o aparecimento de condições ginecológicas crônicas. Apesar desta algia ser bastante incidente e ter impacto negativo na vida de mulheres, terapêuticas para o seu tratamento são pouco elucidadas na literatura. 
Estudo realizado por Paris et al. (2019), que tinha como objetivo avaliar o efeito da bandagem elástica funcional sobre a dor menstrual, verificou redução da dismenorreia, o que significa que esta terapia pode impactar positivamente na melhora dos quadros álgicos da dismenorreia primária.

\section{Exercícios Físicos}

A utilização de exercícios físicos, tanto em ritmos habituais quanto em maior vigorosidade, para redução das dores menstruais demonstraram que a intervenção é aplicável e viável e que os resultados preliminares mostraram evidências de mudanças positivas após a intervenção (A20, A21). O estudo A19 aponta a necessidade de mais estudos para investigar a hipótese de que 0 exercício reduz os sintomas associados à dismenorreia.

Estudo realizado por Silva, Regis e Silva (2017) que buscaram analisar a eficácia dos exercícios físicos no tratamento dos sintomas da dismenorreia primária, constataram que a prática de exercícios físicos contribui positivamente para a redução da sintomatologia e que pode ser considerada uma opção eficaz.

\section{Pilates}

O Método Pilates é uma terapia não farmacológica baseado em movimentos do corpo todo, respiração, concentração, centralização, precisão, ritmo e equilíbrio (MARTINEZ et al., 2018). O estudo A22 apontou que o Pilates é uma opção benéfica para o alívio da dismenorreia, pois além de alívio álgico, os exercícios proporcionaram melhora da flexibilidade muscular, redução da dor, dos desconfortos associados e melhora na qualidade de vida, assim o método pode ser uma terapia simples e de baixo custo para o tratamento sintomático da dismenorreia primária.

Corrobora com este estudo, os resultados da pesquisa de Araújo et al. (2012) que tinham como objetivo comparar a dor menstrual antes e após serem submetidas ao Pilates, e observaram significativa melhora dos sintomas, mostrando-se uma alternativa não farmacológica promissora.

\section{Yoga}

O Yoga traz em si o equilíbrio entre corpo, mente e espírito, constituindo os pilares culturais que foram transmitidos por diversas gerações de mestres e discípulos. A visão ocidentalista do esporte durante muito tempo tem prevalecido apenas como prática corporal, que separa o indivíduo de suas emoções, seus limites e potencialidades, mas tem encontrado na psicologia, na filosofia e práticas orientais, respaldo para o controle de si mesmo (NUNES et al., 2016).

O estudo A24 buscou investigar os efeitos da prática de yoga sobre estudantes com dismenorreia primária, realizando este método uma vez por semana pelo período de doze semanas, como resultado notou-se redução da intensidade da dor menstrual em comparação com o grupo controle. A prática de Yoga pode diminuir o sofrimento pré-menstrual e melhorar a saúde das mulheres (A23). 
Os resultados deste estudo corroboram com a pesquisa de Ulaa et al. (2020) que buscava compreender a eficácia no uso da Yoga para quadros álgicos da dismenorreia primária, seus resultados apontaram que a intervenção proposta foi eficaz na redução dos sintomas.

\section{Estimulação Elétrica Nervosa Transcutânea e Termoterapia}

A Estimulação Elétrica Nervosa Transcutânea (TENS) é comumente usada para reduzir a dor musculoesquelética e para melhorar a função (BUTERA et al., 2018). Os estudos analisados nesta pesquisa apresentaram o uso do TENS isolado e também junto ao uso da termoterapia aplicando-se calor com objetivos terapêuticos, promovendo o alívio da dismenorreia e foi considerado uma terapia não farmacológica efetiva e segura (A25, A26, A27, A28).

Estudos realizados por Torrilhas et al. (2017) e Oliveira e Cirqueira (2019) referem que o uso da TENS é capaz de reduzir os sintomas causados pela dismenorreia primária, melhorando a qualidade de vida das mulheres, 0 demonstra que esta terapia é um importante recurso não invasivo.

\section{Raios Infravermelhos}

O estudo A29 aborda os efeitos dos raios infravermelhos em mulheres que apresentavam dismenorreia usando um cinto abdominal de raio infravermelho durante um ciclo menstrual até o final do período menstrual, enquanto o grupo controle usava compressas quentes. Os resultados deste estudo indicam que o raio de Light Emitting Diode (LED) infravermelho foi eficaz na redução e duração da dor menstrual, assim os raios infravermelhos próximos ao corpo podem ser usados para aliviar a dor menstrual e melhorar a qualidade de vida dos pacientes com dismenorreia.

\section{CONSIDERAÇÕES FINAIS}

A dismenorreia primária é apontada como um distúrbio álgico em região suprapúbica associada também a outros sintomas, que ocorrem no período menstrual. Este desconforto provoca redução da qualidade das atividades de vida diária, atenuação da produtividade laboral e absenteísmo, constituindo-se um problema de saúde pública, o que torna imprescindível a busca por tratamentos eficientes.

De acordo com os estudos, após a realização da terapia, houve redução na intensidade dos sintomas álgicos da dismenorreia, o que impacta positivamente na melhora da qualidade de vida das mulheres. Os métodos não farmacológicos relevantes para a redução deste desconforto foram: acupressão; acupuntura; moxabustão; aromaterapia; massagem aromaterapêutica; massagem terapêutica, bandagem elástica funcional, exercícios físicos, pilates, yoga, TENS, termoterapia e uso de raios infravermelhos.

Nesse sentido, é de grande importância que todos os profissionais de saúde conheçam estes métodos, pois possibilita a abordagem integral, orientação eficiente e assertiva. 
Como limitação desta revisão, salienta-se que a busca dos estudos foi realizada em apenas duas bases de dados e que não foi abordado possíveis efeitos colaterais das terapias apresentadas, assim sugere-se novos estudos acerca da temática.

\section{REFERÊNCIAS}

ABARAOGU, U. O.; TABANSI-OCHUOGU, C. S. As Acupressure Decreases Pain, Acupuncture May Improve Some Aspects of Quality of Life for Women with Primary Dysmenorrhea: A Systematic Review with Meta-Analysis. Journal of Acupuncture and Meridian Studies, v. 8, n. 5, p. 220-228, 2015.

ABARAOGU, U. O.; IGWE, S. E.; TABANSI-OCHIOGU, C. S. Effectiveness of SP6 (Sanyinjiao) acupressure for relief of primary dysmenorrhea symptoms: A systematic review with meta- and sensitivity analyses. Complementary Therapies in Clinical Practice, v. 25, p. 92-105, 2016.

ALVES, T. P. et al. Dismenorreia: Diagnóstico e Tratamento. Revista Científica da Faculdade de Educação e Meio Ambiente, v. 7, n. 2, p. 1-12, 2016.

ARAUJO, L. M. et al. Diminuição da dor em mulheres com dismenorreia primária, tratadas pelo método Pilates. Revista Dor, v. 13, n. 2, p. 119-123, 2012.

ARMOUR, M. et al. The role of treatment timing and mode of stimulation in the treatment of primary dysmenorrhea with acupuncture: An exploratory randomised controlled trial. PLoS One, v. 12, n. 7, e0180177, 2017.

ARMOUR, M.; SMITH, C. A. Treating primary dysmenorrhoea with acupuncture: a narrative review of the relationship between acupuncture 'dose' and menstrual pain outcomes. Acupuncturein Medicine, v. 34, n. 6, p. 416-424, 2016.

AZIMA, S. et al. Comparison of the Effect of Massage Therapy and Isometric Exercises on Primary Dysmenorrhea: A Randomized Controlled Clinical Trial. Journal of Pediatric and Adolescent Gynecology, v. 28, n. 6, p. 486-491, 2015.

$\mathrm{BAI}, \mathrm{H}$;; BAI, H.; YANG, Z. Effect of transcutaneous electrical nerve stimulation therapy for the treatment of primary dysmenorrheal. Medicine (Baltimore), v. 96, n. 36, e7959, 2017.

BARACAT, E. C.; FONSECA, A. M.; BAGNOLI, V. R. Terapêutica Clínica em Ginecologia. São Paulo: Manole, 2015.

BAVIL, D. A. et al. Comparison of lifestyles of young women with and without primary dysmenorrhea. Electronic Physician Journal, v. 8, n.3, p. 2107-2114, 2016. 
BROWN, J.; BROWN, S. Withdrawn: Exercise for dysmenorrhoea. Cochrane Database of Systematic Reviews, v. 2, CD004142, 2017.

BUTERA, K. A. et al. Prolonged Reduction in Shoulder Strength after Transcutaneous Eletrical Nerve Stimulation Treatment of Exercise Induced Acute Muscle Pain. Pain Practice: the Official Journal of World Institute of Pain, v. 18, n. 8, p. 954-968, 2018.

CAMPOS, S. et al. Efeito da bandagem elástica funcional no equilíbrio dinâmico: Estudo piloto. XX Encontro Latino Americano de Iniciação Científica, XVI Encontro Latino Americano de Pós-Graduação e VI Encontro de Iniciação à Docência - Universidade do Vale do Paraíba. 2016.

CHA, N. H.; SOK, S. R. Effects of Auricular Acupressure Therapy on Primary Dysmenorrhea for Female High School Students in South Korea. Journal of Nursing Scholarship, v. 48, n. 5, p. 508-516, 2016.

CHEN, H. et al. Effects of acupressure on menstrual distress and low back pain in dysmenorrheic young adult women: an experimental study. Pain Management Nursing, v. 16, n. 3, p. 188-197, 2015.

DEHKORDI, Z. R.; BAHARANCHI, H. F. S.; BEKHRADI, R. Effect of lavender inhalation on the symptoms of primary dysmenorrhea and the amount of menstrual bleeding: A randomized clinical trial. Complementary Therapies in Medicine, v. 22, n. 2, p. 212-219, 2014.

DIAS, J.; MELO, N. J. Tratamento da tensão pré-menstrual e as manifestações emocionais com acupuntura. 2014. 46f. Monografia (Especialista em Acupuntura) - Universidade de Mogi das Cruzes, São Paulo, 2014.

FONSECA, J. M. A. et al. A influência do método Pilates na flexibilidade muscular, sintomas e qualidade de vida em mulheres com dismenorreia primária. Scientia Medica, v. 26, n. 2, ID23052, 2016.

GAO, J. et al. The effect of moxibustion on alleviating menstrual pain in a population of Young nursing students: A prospective randomized cross-over pilot study. Complementary Therapies in Medicine, v. 23, n. 6, p. 773-781, 2015.

GNATTA, J. R. et al. Aromaterapia e enfermagem: concepção histórico-teórica. Revista da Escola de Enfermagem da USP, v. 50, n. 1, p. 130-136, 2016.

IGWEA, S. E.; TABANSI-OCHUOGU, C. S.; ABARAOGU, U. O. TENS and heat therapy for pain relief and quality of life improvement in individuals with primary dysmenorrhea: A systematic review. Complementary Therapies in Clinical Practice, v. 24, p. 86-91, 2016. 
KANNAN, $P$. et al. Vigorous exercises in the management of primary dysmenorrhea: a feasibility study. Disability and Rehabilitation, v. 37, n. 15, p. 1334-1339, 2015a.

KANNAN, P. et al. Menstrual pain and quality of life in women with primary dysmenorrhea: Rationale, design, and interventions of a randomized controlled trial of effects of a treadmill-based exercise intervention. Contemporary Clinical Trials, v. 42, p. 81-89, 2015b.

LAURETTI, G. R. et al. The New Portable Transcutaneous Electrical Nerve Stimulation Device Was Efficacious in the Control of Primary Dysmenorrhea Cramp Pain. Neuromodulation, v. 18, n. 6, p. 522-526, 2015.

LEE, B. et al. Efficacy of the device combining high-frequency transcutaneous electrical nerve stimulation and thermotherapy for relieving primary dysmenorrhea: a randomized, single-blind, placebo-controlled trial. European Journal of Obstetrics \& Gynecology and Reproductive Biology, v. 194, p. 58-63, 2015.

LEE, J.; KIM, K. Effect of near-infrared rays on female menstrual pain in Korea. SciNursing \& Health Sciences, v. 19, n. 3, p. 366-372, 2017.

LIMA, I. D. T. G. Efeitos da massagem terapêutica e aromaterapia: Revisão de literatura. 2016. 17f. Trabalho de Conclusão de Curso (Graduação em Fisioterapia) - Universidade Estadual da Paraíba, Campina Grande, 2016.

LIU, C. Z. et al. A randomized controlled trial of single point acupuncture in primary dysmenorrhea. Pain Medicine, v. 15, n. 6, p. 910-920, 2014.

LUMSDEN, M. A.; ILIODROMITI, S. Avaliação da dismenorreia. BMJ Best Practice. 2015. Disponível em: <http://brasil.bestpractice.bmj.com/bestpractice/monograph/420.html>. Acesso em: 13 ago. 2018.

MARTINEZ, J. A. R. et al. Equilíbrio corporal, mobilidade e força muscular respiratória de idosas praticantes do método Pilates. Revista Fisioterapia e Pesquisa, v. 8, n. 1, p. 8-15, 2018.

MELNYK, B. M.; FINEOUT-OVERHOLT, E. Evidence-based practice in nursing \& healthcare: a guide to best practice. 4. ed. Philadelphia: Wolters Kluwer Health, 2019.

MENDES, K. D. S.; SILVEIRA, R. C. C. P.; GALVÃO, C. M. Revisão integrativa: método de pesquisa para a incorporação de evidências na saúde e na enfermagem. Texto \& contexto enfermagem [internet], v. 17, n. 4, p. 758-764, 2008.

NUNES, J. M. O. et al. Dismenorreia em universitárias. Revista Brasileira em Promoção da Saúde, v. 26, n. 3, p. 381-386, 2013. 
OLIVEIRA, M. M.; CIRQUEIRA, R. P. Eficácia da eletroterapia na dismenorreia: revisão de literatura. ID on line Revista de Psicologia, v. 13, n. 43, p. 448-454, 2019.

PARIS, B. et al. Avaliação do efeito da bandagem elástica funcional sobre a dismenorreia primária em jovens universitárias. Fag Journal of Health, v. 1, n. 1, p. 167-174, 2019.

PINHO, L. E. et al. Terapia manual no tratamento da dismenorreia primária: revisão sistemática. Revista Pesquisa em fisioterapia, v. 7, n. 2, 2017.

PIVETA, A. Ensino e aprendizagem sobre medicina Tradicional chinesa e técnicas de Acupressão como possibilidade de cuidado em saúde. 2015. 94f. Dissertação (Mestrado em Enfermagem) - Universidade de Santa Maria, Santa Maria, 2015.

SHAHR, H. S. A. et al. The effect of self-aromatherapy massage of the abdomen on the primary dysmenorrhoea. Journal of Obstetrics and Gynaecology, v. 35, n. 4, p. 382-385, 2015.

SANTOS, A. F, et al. Métodos não farmacológicos utilizados para o alívio da dismenorreia: Revisão Integrativa. Journal of Health Connections, v. 1, n. 1. p. 19-32, 2017.

SILVA, J. R. A.; REGIS, T. S.; SILVA, A. Q. G. Exercício físico como terapia não farmacológica no tratamento da dismenorreia primária: uma revisão sistemática. Revista Pesquisa em Fisioterapia, v. 7, n. 4, p. 470-480, 2017.

SMITH, C. A. et al. Acupuncture for dysmenorrhoea. Cochrane Database of Systematic Reviews, v. 4, n. CD007854, 2016.

SMITH, R. P.; KAUNITZ, A. M. Primary dysmenorrhea in adult women: Clinical features and diagnosis. In: post, T.W. Ed. Up To Date. Waltham, MA: Up To Date, 2017.

STALLBAUM, J. H. et al. Efeitos da bandagem funcional elástica sobre a dismenorreia primaria em universitárias. Fisioterapia Brasil, v. 17, n. 6, p. 518525, 2016.

SUT, N.; KAHYAOGLU-SUT, H. Effect of aromatherapy massage on pain in primary dysmenorrhea: A meta-analysis. Complementary Therapies in Clinical Practice, v. 27, p. 5-10, 2017.

TORRILHAS, M. C. et al. Estimulação elétrica nervosa transcutânea na dismenorreia primária em mulheres jovens. Revista de Atenção à Saúde, v. 15, n. 54, p. 61-66, 2017. 
TSAI, S. Effect of Yoga Exercise on Premenstrual Symptoms among Female Employees in Taiwan. International Journal of Environmental Research and Public Health, v. 13, n. 7, p. 721, 2016.

ULAA, M. A. et al. Proceedings of the 1st International Conference on Science, Health, Economics, Education and Technology (ICoSHEET 2019). Advances in Health Sciences Research, v. 27, 2020.

ULRICH, W. Livre-se das dores pela acupuntura e acupressura. Tradução de Maria Madalena Würth Teixeira. Rio de Janeiro: Ediouro, 1980.

UYSAL, M. et al. Investigating the effect of rose essential oil in patients with primary dysmenorrhea. Complementary Therapies in Clinical Practice, v. 24, p. 45-49, ago. 2016.

VILLAHERMOSA, D. I. M. et al. Influence of acupuncture on the outcomes of in vitro fertilisation when embryo implantation has failed: a prospective randomised controlled clinical trial. Acupuncture in Medicine, v. 31, n. 2, p. 157-161, 2013.

WOO, H. L. et al. The efficacy and safety of acupuncture in women with primary dysmenorrhea: A systematic review and meta-analysis. Medicine (Baltimore), v. 97, n. 23, e11007, 2018.

$\mathrm{XU}, \mathrm{Y}$. et al. Effects of acupoint-stimulation for the treatment of primary dysmenorrhoea compared with NSAIDs: a systematic review and meta-analysis of 19 RCTs. BMC Complementary and Alternative Medicine, v. 17, n. 1, p. 436, 2017.

YAMAMURA, Y. Acupuntura tradicional: a arte de inserir. 2. ed. São Paulo: Roca, 2001.

YANG, J. et al. Use of moxibustion to treat primary dysmenorrhea at two interventional times: study protocol for a randomized controlled trial. Trials, v. 16, n. 35, 2015.

YANG, N.; KIM, S. Effects of a Yoga Program on Menstrual Cramps and Menstrual Distress in Undergraduate Students with Primary Dysmenorrhea: A Single-Blind, Randomized Controlled Trial. Journal of Alternative and Complementary Medicine, v. 22, n. 9, p. 732-738, 2016. 